\title{
Tsunami wave generation by the eruption of underwater volcano
}

\author{
Y. Egorov \\ Institute for Advanced Studies, Vienna, Austria \\ Received: 16 October 2006 - Revised: 8 January 2007 - Accepted: 8 January 2007 - Published: 19 January 2007
}

\begin{abstract}
Eruption of volcanoes represents one of important origins of tsunami waves and is responsible for most catastrophic tsunami (Krakatau, 1883; Thira, BC). The products of volcano eruption include solids, liquids (lava) and gases. The present article presents hydrodynamic model of relatively slow process of eruption, with domination of liquids. The process of underwater eruption of lava causes the disturbance of ocean free surface. The standard formulation of hydrodynamic problem for incompressible fluid in cylindrically symmetric layer of with rigid bottom and free surface with local hydrodynamic source (volcano) is used. This problem is solved by constructing Green function using methodology of Sretenskij. The solution is obtained in the form of an integral and depends on the dynamics of eruption. Real data show that some volcanoes can erupt several millions of tons of lava during several dozens of seconds (Bezimjannij, Kamchatka). The long waves are more efficiently generated by larger $T$ : these tsunamis can have smaller initial perturbations of free surface, but the waves are long and can transmit their energy over longer distances.
\end{abstract}

\section{Introduction}

About 6 to $15 \%$ of al tsunami emerge from non-seismic origins (Pelinovskij, 1982). The greater share of them come from the eruption of volcanoes. Among about 100 events of volcanic origin, $29 \%$ are caused by subaquatic volcanic explosions (Belousov et al., 2000). These eruptions can create catastrophic tsunami waves. For example, after the eruption of Krakatau volcano in 1883 the wave height upon reaching the sea shore was 45 meters. The subaquatic volcanic explosion in Karymskoe lake (Kamchatka, 1996) presented an interesting tsunami case (Belousov et al., 2000).

Correspondence to: Y. Egorov

(yegorov@ihs.ac.at)
It is well known that the products of volcano eruption include solids, liquids (lava) and gases (Taziev, 1963). The composition of these components differ with cases and with evolution, as well as the speed of eruption (Woods et al., 1995). Substantial research is done about tsunamis generated by landslides of volcanoes and islands (Bardet et al., 2003; Tinti et al., 2001). In fact, many volcanoes are partly above the sea level, and the movement of solids can play substantial role here. However, there are also hidden volcanoes on the sea bottom (including Mediterranean sea, that has been considered as the area of low tsunami danger), and for them hydrodynamic processes in tsunami generation should dominate. In the present article the focus is on hydrodynamic model of generation of tsunami waves, which is related to the slow process of eruption.

The case of fast eruption has some similarity the underwater explosion, that was studied in more substantially in different applications (Ivanov and Koroljov, 1986; Pelinovskij, 1982; Le Mehaute, 1971). However, as it was shown in Pelinovskij (1982), acoustic (or fast eruption) model generates rather weak field at the substantial distances from the source. This differs from natural observations of tsunami: they practically always have substantial field at large distances, due to involvement of long gravity waves into the propagation process. If we turn to the data of the eruption dynamics provided by volcanologists, it is possible to find out that many temporal patterns of eruptions (and their spectra) differ from those typical for explosions; they are characterized by having mostly components with lower frequencies (Brechovskih, 1974).

In the present model it is assumed that eruption takes place for underwater volcano with the dominance of liquid components. Since the process is assumed to be relatively slow (that is, it has characteristic time above several seconds) it is possible to neglect the compressibility of water and to consider purely hydrodynamic model. The formal exposition of the model follows Egorov (1990).

Published by Copernicus GmbH on behalf of the European Geosciences Union. 


\section{Model formulation}

\subsection{The core equations}

Consider the following hydrodynamic model written for the velocity potential of small elements of water $\phi$ :

$\Delta \phi=-4 \pi q(t) \delta\left(\boldsymbol{X}-\boldsymbol{X}_{0}\right)$.

Here $\Delta$ denotes the operator of Laplace, while the right side of Eq. (1) has nonstationary hydrodynamic source located at the point $\boldsymbol{X}=\boldsymbol{X}_{0}$. While the water area of the validity of this equation has complex shape (that contains free surface and uneven bottom), it is possible to concentrate on the problem with cylindrical symmetry, where the ocean depth does not depend on any of horizontal coordinates. Mathematical simplicity is not the unique reason for such choice. As it was shown in Pelinovskij (1982), the method of wave ray tubes can be also used for tsunami waves. Within the ray the variation of bottom depth in the direction orthogonal to ray is typically small, and the main evolutionary effect comes from ray divergence, which is similar to one taking place in the model with cylindrical symmetry. The bottom is described by the surface $z=0$, while the unperturbed free surface is given by $z=D$. The coordinate $r$ characterizes the horizontal distance from the crater centre, and due to assumed radial symmetry the angular variable does not enter equations, and thus can be omitted.

In hydrodynamics, the boundary conditions at the free surface are typically nonlinear (Sretenskij, 1977). This nonlinearity is very important for the tsunami waves, if they have substantial wave amplitudes. If the eruption is substantial, i.e. the ratio of wave height to the ocean depth near crater is not small, the use of nonlinear boundary condition becomes important. At the same time, mathematical simplifications are required for the construction of analytically tractable base model. That is why further derivations will be based on linear condition on the free surface (see Sretenskij, 1977):

$$
\frac{\partial^{2} \phi}{\partial t^{2}}+g \frac{\partial \phi}{\partial z}=0 \quad(z=D) .
$$

In the approximation of the rigid bottom (typical for hydrodynamics), there is Neumann boundary condition there:

$$
\frac{\partial \phi}{\partial z}=0 \quad(z=0) .
$$

Since all typical scales of length in tsunami models are substantially larger than the crater size, it is possible to approximate the one with a point source, located at $(z=\epsilon, r=0)$. In this case we have the source at the distance $\epsilon$ from the bottom level. While the real bottom topography near crater is more complex, this mathematical model can still be used. Being a rough approximation for the investigated physical processes, it still captures those assumptions that are most important for relevant description of tsunami generation. The model presented below does not pretend to fully describe the complex processes. Its main goal is to detect those parameters that influence tsunami wave generation and to estimate tsunami intensity as the function of these parameters. It will be shown that the characteristic parameters that are linked with the dynamics of eruption play an important role for the parameters of tsunami wave at large distances from the volcano.

\subsection{The physical interpretation of hydrodynamic source}

In the symmetric 3-dimensional space, the source with debit $q(t)$ generates the field $\phi_{0} q(t) / R$ in some neighbourhood of the source point. In hydrodynamic models, liquid is assumed to be incompressible, and thus acoustic waves with finite speed of propagation cannot be described properly. But since this acoustic speed is high for water, being about $1500 \mathrm{~m} / \mathrm{s}$, for relatively slow eruption processes, lasting above several seconds, an assumption of an infinite acoustic speed does not give substantial error up to the distances of about $1 \mathrm{~km}$. However, at these distances the assumption of 3-dimensional symmetry (point neighbourhood in $R^{3}$ ) is no longer valid, since the wave touches either bottom or free surface.

Due to Gauss theorem, which says that the surface integral from the scalar product of the potential gradient and the normal vector to surface element equals to the source inside multiplied by $4 \pi$, we can say that the volume of liquid $w$ passing through the sphere surrounding the source (crater) equals to

$w=\Delta t \int_{S} v_{n} d S=\Delta t 4 \pi q(t)$.

The final formulation of hydrodynamic problem requires addition of initial conditions. Assuming water to be at rest before the beginning of eruption, we can set all deviations and velocities equal to zero. This requires setting:

$\phi(r, z, t=0)=0 ; \quad \partial \phi(r, z, t=0) / \partial t=0$.

\section{Solution to the problem about hydrodynamic field of non-stationary source}

Consider the following initial-boundary problem (here $\Delta$ is 2-dimensional Laplace operator in polar coordinates):

$$
\begin{array}{rr}
\Delta \phi+\frac{\partial^{2} \phi}{\partial z^{2}}=-4 \pi q(t) \delta(r) \delta\left(z-z_{0}\right), \\
\frac{\partial^{2} \phi}{\partial t^{2}}+g \frac{\partial \phi}{\partial z}=0 & (z=D), \\
\frac{\partial \phi}{\partial z}=0 & (z=0), \\
\phi(r, z, t=0) & =0, \\
\partial \phi(r, z, t=0) / \partial t & =0 .
\end{array}
$$

It is assumed that the function $4 q(t)$ is finite and differs from zero only inside the interval $[0, T]$. This can be interpreted as the finiteness of the duration of eruption. We use cylindrical 
coordinates $(r, z)$ and radial symmetry around the point of eruption ( $z$ - vertical coordinate, $r$ - radial coordinate), $t$ is time, $\phi$-hydrodynamic potential (its gradient gives velocity of particles, $z=D$ is non-perturbed free surface of the ocean, while $z=0$ describes the shape of the bottom.

Hydrodynamic source, described by the product of Dirak functions $\delta$, represents the model of local source (eruption). For slow eruptions the wavelength of tsunami wave is much larger than the scale of crater, and the assumption of localized source is asymptotically valid. It is important to mention a couple of other assumptions for this model. First of all, any volcano has a conic form and creates local heterogeneity in ocean relief, which is not accounted for here. Another assumption is about radial symmetry of the ocean bottom. In reality, bottoms have two-dimensional heterogeneity, but it is not so important for our results. As it was shown in Pelinovskij (1982), energy of tsunami waves is propagating along the ray tubes, and inside each (narrow) tube the depth variation along the coordinate orthogonal to the ray can be neglected (we use the mean in local cross-section).

\subsection{Details of mathematical solution to the model}

Now the model is fully formalized, and it can be solved using the method of Green function (see Sretenskij, 1977). The solution to such problem is sought in the form of source, its reflected image from the bottom and some additional potential $\psi$ :

$$
\begin{array}{r}
\phi(r, z, t)=\frac{q(t)}{\left[r^{2}+\left(z-z_{0}\right)^{2}\right]^{1 / 2}}+ \\
\frac{q(t)}{\left[r^{2}+\left(z+z_{0}\right)^{2}\right]^{1 / 2}}+\psi(r, z, t) .
\end{array}
$$

Substituting this decomposition (11) into the initial problem (6-10), we get the problem for additional potential $\psi$ :

$$
\begin{aligned}
& \frac{\partial}{r \partial r}\left[r \frac{\partial \psi}{\partial r}\right]+\frac{\partial^{2} \psi}{\partial z^{2}}=0, \\
& \frac{\partial \psi}{\partial z}=0 \quad(z=0), \\
& \frac{\partial^{2} \psi}{\partial t^{2}}+g \frac{\partial \psi}{\partial z}=-\left(\frac{\partial^{2} \phi_{1}}{\partial t^{2}}+g \frac{\partial \phi_{1}}{\partial z}\right) \quad(z=D),
\end{aligned}
$$

where $\phi_{1} \equiv \phi-\psi$.

The requirement of limited solutions for all values of arguments leads to the search of the solution in cylindrical coordinates as the superposition of Bessel functions of $r$ and hyperbolic functions of $z$, that also satisfy border conditions:

$\psi(r, z, t)=\int_{0}^{\infty} C(\lambda, t) J_{0}(\lambda r) \operatorname{ch}[\lambda z] d \lambda$,

where $J_{0}$ is the Bessel function of zero order and $\lambda$ is an arbitrary parameter. Substitution of anzatz leads to the following differential equation for $C(\lambda, t)$ :

$$
\begin{array}{r}
\frac{d^{2} C}{d t^{2}}+g \lambda \operatorname{th}(\lambda D) C=F(t, \lambda), \\
F(t, \lambda) \equiv 2 e^{-\lambda D} \frac{\operatorname{ch}(\lambda \epsilon)}{\operatorname{ch}(\lambda D)}\left[g \lambda q(t)-q^{\prime \prime}(t)\right] .
\end{array}
$$

The solution to differential equation is the sum of its general and particular solutions

$$
\begin{array}{r}
C(\lambda, t)=A(\lambda) \cos (\omega t)+B(\lambda) \sin (\omega t)+ \\
\frac{1}{\omega} \int_{0}^{t} F(\tau, \lambda) \sin [\omega(t-\tau)] d \tau,
\end{array}
$$

where $\omega \equiv \sqrt{g \lambda t h(\lambda D)}$.

The initial conditions allow to define

$$
\begin{gathered}
A(\lambda)=-\frac{2 q(0) \exp (-\lambda z) \operatorname{ch}(\lambda \epsilon)}{\operatorname{ch}(\lambda z)}, \\
B(\lambda)=-\frac{2 q^{\prime}(0) \exp (\lambda z) \operatorname{ch}(\lambda \epsilon)}{\omega \operatorname{ch}(\lambda z)},
\end{gathered}
$$

and thus to find the unique solution to the problem.

The general solution that can satisfy different initial condition then has the following form:

$$
\begin{gathered}
\phi(r, z, t)=\frac{q(t)}{\left[r^{2}+(z-\epsilon)^{2}\right]^{1 / 2}}+\frac{q(t)}{\left[r^{2}+(z+\epsilon)^{2}\right]^{1 / 2}}+ \\
\int_{0}^{\infty} J_{0}(\lambda r) \operatorname{ch}(\lambda z)[A(\lambda) \cos (\omega t)+B(\lambda) \sin (\omega t)] d \lambda+ \\
2 \int_{0}^{\infty} \frac{d \lambda}{\omega} \int_{0}^{t} d \tau e^{-\lambda D} \frac{\operatorname{ch}[\lambda \epsilon]}{\operatorname{ch}[\lambda D]}\left[g \lambda q(\tau)-\frac{d^{2} q(\tau)}{d \tau^{2}}\right] * \\
\sin [\omega(t-\tau)] \operatorname{ch}[\lambda z] J_{0}(\lambda r), \quad \omega \equiv \sqrt{g \lambda t h(\lambda D)} .(21)
\end{gathered}
$$

\section{The process of tsunami generation}

The details of eruption processes are complex (Woods et al., 1995). Ideally, we should know the typical intensity as a function of time. But in most cases only the duration of eruption $T$ and the total mass of erupted substance $W$ are measured. It is natural to model the dynamics of eruption as a smooth process, when in the first period the intensity is increasing, reaching maximum and then declining. ${ }^{1}$ Assume that the eruption occur during time interval $T$ and that its intensity varies in time as:

$q(t)=\frac{W}{2 \pi T} \sin ^{2} \frac{\pi t}{T}, \quad(0 \leq t \leq T)$.

\footnotetext{
${ }^{1}$ Working with non-smooth functions creates additional mathematical problems, but gives no gain in our understanding of the process, since they contribute to high-frequency spectrum which does not affect generation of tsunami wave, which has low-frequency spectrum.
} 
This class of functions is smooth, and satisfies the initial conditions:

$q(0)=0, \quad q^{\prime}(0)=0$.

Then from the formulae $(19,20,23)$ we get:

$A(\lambda) \equiv 0, \quad B(\lambda) \equiv 0$.

The initial shift of the water surface can be calculated from the potential $\phi$ using formula

$h(r ; T)=\frac{1}{g} \frac{\partial \phi(r, 0, t)}{\partial t}$.

After the end of eruption the disturbance of free surface can be described by the integral expression ${ }^{2}$ :

$$
\begin{array}{r}
h(r, t)=\frac{\pi W}{g T^{3}} * \\
\int_{0}^{\infty} d \lambda J_{0}(\lambda r) \operatorname{ch}[\lambda \epsilon] \exp (-\lambda D) \frac{\sin [\omega t]-\sin [\omega(t-T)]}{\omega(\lambda)} .
\end{array}
$$

This formula gives the efficient tsunami-generating zone, and further evolution of tsunami wave can be analysed as autonomous process, independent from eruption.

\subsection{Asymptotic analysis}

Since the ocean depth $D$ (average near volcano) is the characteristic scale, while the characteristic speed is $c=\sqrt{g D}$, the characteristic time scale is $t_{0}=\sqrt{D / g}$. Now it is possible to introduce scale-free variables

$\rho=r / D, \quad \tau=t \sqrt{g / D}$.

Besides that, more scale-free parameters can be introduced:

$\kappa=T \sqrt{g / D}, \quad V=W / D^{3}, \quad \gamma=\epsilon / D$.

The scale-free function $H=h / D$ describes the field of local deviations of free surface:

$$
\begin{array}{r}
H(\rho, \tau)=2 \pi V \kappa^{-3} \int_{0}^{\infty} d \mu J_{0}(\mu \rho) e^{-\mu} G(\mu, \tau, \kappa, \gamma), \\
G \equiv \frac{2 \sin [\omega \kappa / 2] \cos [\omega(\tau-\kappa / 2)]}{\omega} \operatorname{ch}[\mu \gamma] \\
\omega(\mu) \equiv \sqrt{\mu t h(\mu)}
\end{array}
$$

Let us consider the shape of wave $H$ at the end of hypothetical eruption, i.e. for $\tau=\kappa$. Then,

$H(\rho, \tau)=2 \pi V \kappa^{-3} \int_{0}^{\infty} d \mu J_{0}(\mu \rho) e^{-\mu} \operatorname{ch}(\gamma \mu) \frac{\sin (\kappa \omega)}{\omega}(29)$

\footnotetext{
${ }^{2}$ If there are several eruptions, we focus on a particular subperiod of peak intensity. Here the contribution of the term $\phi_{1}$ disappears since $q(t)=0$, for $t>T$.
}

Expanding this integrand into Taylor series, we can find asymptotics for $\kappa \rightarrow 0$ :

$$
\begin{array}{r}
H(\rho, \tau)=\pi V \kappa^{-2}\left[\frac{1}{\rho^{2}+(1-\gamma)^{2}}+\right. \\
\left.\frac{1}{\rho^{2}+(1+\gamma)^{2}}\right]\left(1+O\left(\kappa^{2}\right)\right) .
\end{array}
$$

This expression shows that initial perturbation of free surface decays inversely proportional to the horizontal distance.

Since the hydrodynamic model neglects the finiteness of sound propagation speed in wave $c_{0}$, it is asymptotically valid only in the limited area $R_{0} \leq c_{0} T$. Since for the whole ocean we have the global estimate

$c_{0}>>\sqrt{g D}$,

we have the inequality:

$\delta \equiv \frac{R_{0}}{D} \approx \frac{c_{0} T}{D}>>\frac{\sqrt{g D} T}{D}=\kappa$.

Thus parameter $\delta$ is rather large even for small values of $\kappa$. This means that long surface waves can be efficiently generated by volcano eruption for many regimes of its activity.

Numerical calculations of the solution show that at $R / D=10$, the wave shape does not depend much on the variation of parameter $\kappa$ between 0.1 and 3 . In all cases positive half-wave is formed, followed by oscillations.

Consider a hypothetical example of bottom volcano at the depth $1 \mathrm{~km}(D=1 \mathrm{~km}, \epsilon=0)$, with the eruption duration of $T=10 \mathrm{~s}$ and the volume of erupted substance $W=10^{6} \mathrm{~m}^{3}$. Then at the distance $10 \mathrm{~km}$ we can expect the wave with the height of $15 \mathrm{~m}$ and the half-period of about $60 \mathrm{~s}$. For such a wave the Ursell parameter $U=h \lambda^{2} / D^{3}$ is of order one. Hence, further evolution is driven by both nonlinear ands dispersion effects, and such waveform can lead to the emergence of a soliton. In a channel (or ray tube of constant width) solitons can propagate without decay, while radially symmetric waves with $U=O(1)$ have the decay in height as $R^{-1 / 2}$ (Pelinovskij, 1982).

\subsection{Conditions of model applicability}

It is useful to provide some energetic estimations. Let $S$ denotes the area of crater cross-section, $v$ the density of erupted substance and $v$ - the speed of erupted lava. Since we always have the balance equation $v=W /(T S)$, the kinetic energy $E_{k}$ of eruption is given by the formula

$E_{k}=\frac{v W^{3}}{2 T^{2} S^{2}}$.

If the speed $V$ is too high (fast eruption), the erupted lava will go to the air above the free surface, the effective radius of initial perturbation of water surface will be small, and efficient generation of long waves will not take place. The model is 
not valid, since such stream of lava cannot be treated as hydrodynamic source. The condition of lava exiting to air is the following:

$V \geq \sqrt{2 g(D-\epsilon)}$

The present model is valid if two conditions are satisfied (sufficiently low velocity of eruption and sufficiently long time of eruption):

$$
V<<\sqrt{2 g(D-\epsilon)}, \quad T>>\frac{W}{S \sqrt{2 g(D-\epsilon)}} .
$$

\subsection{Wave height in crater zone}

The initial elevation of water (wave height) over volcano zone $(r=0)$ can be calculated asymptotically for low $T$; notations $h=D, z_{0}=D-\epsilon$ are used)(see Pelinovskij, 1996, p.56):

$h_{0}=\frac{2 \pi W}{g T^{2}} \frac{h}{\left|z_{0}\right|\left(\left|z_{0}\right|+2 h\right)}$.

It is useful to make some estimations. Assuming ocean depth $1 \mathrm{~km}$ and volcano located at its bottom $\left(z_{0}=h=1(\mathrm{~km})\right)$, the eruption volume $W=10^{6} \mathrm{~m}^{3}$ and the duration $T=10 \mathrm{~s}$, the initial shift of water over crater would be about $2 \mathrm{~m}$. If we assume that the erupted liquid has the density of water, the erupted mass is $1 \mathrm{mln}$. tons. These parameters are not exotic. For example, volcano Bezimjannij (Kamchatka) has erupted several millions of tons of lava during several dozens of seconds (Taziev, 1963).

What is the diameter of efficient water disturbance? If we spread $1 \mathrm{mln}$. cubic meters of water as a layer $2 \mathrm{~m}$ high, it will cover only $0.5 \mathrm{sq} . \mathrm{km}$, too small area to efficiently generate long tsunami wave in deep ocean. In reality, those volcanoes that have generated catastrophic tsunamis, were located much closer to surface, and in some cases have been partly above it. For example, Krakatau volcano before its eruption in 1883 represented a caldera of several islands slightly above sea level. Santorini volcano in Greece has similar structure. For these cases it is more natural to assume $z_{0}=100(\mathrm{~m})$ and $h=200(\mathrm{~m})$, and this gives (for the same values of $W$ and $T$ ) much higher initial elevation in the centre: $\eta_{0}=25(\mathrm{~m})$. With the speed of long gravity waves, $\sqrt{g h}$, being $45 \mathrm{~m} / \mathrm{s}$ on the shelf $200 \mathrm{~m}$ deep, the signal would propagate about $450 \mathrm{~m}$ during the eruption time, creating the area of almost 1 sq.km of perturbed water. This is already within the limit of long waves. Longer $T$ would make generation process even more efficient, although the initial amplitude of these waves would be smaller. While all volcanoes can be responsible for tsunami waves in the local zone, the most dangerous case is when long wave is generated: it can pass many thousands $\mathrm{km}$.

\section{Conclusions}

1. The suggested hydrodynamic mechanism of tsunami generation by volcano eruption allows to describe the observed scales of tsunami waves. Some asymptotic estimations show that for typical parameters of observed surface eruptions, underwater volcanoes can generate catastrophic waves.

2. For the typical parameters of erupted mass and duration, the initial perturbation of the ocean level over underwater volcano is substantial. Long waves are generated more efficiently when volcano is located on shelf and not on deep water. But this is typical situation with volcanoes: those which created catastrophic tsunamis in the past had craters close to the sea level.

3. Volcanoes represent an important source of tsunamis. Understanding the mechanisms of wave generation can help to deal with hazard problems. This is especially important in the areas with limited seismic activity. For example, at present time the risk of tsunamis in Mediterranean basin is underestimated due to neglection of volcano-generated tsunamis.

Edited by: E. Pelinovsky

Reviewed by: V. Maximov and S. Tinti

\section{References}

Bardet, J.-P., Synolakis C. E., Davies, H. L., Imamura, F., and Okal, E. A.: Landslide tsunamis: recent findings and research directions, Pure Appl. Geophys., 160, 1793-1809, 2003.

Belousov, A., Voight, B., Belousova, M., and Muravyev, Y.: Tsunami generated by subaquatic volcanic explosions: unique data from 1996 eruption in Karymskoe lake, Kamchatka, Russia, P. Appl. Geophys., 157, 1135-1143, 2000.

Brehovskih L. M. (Ed.): Acoustics of the Ocean, 693 p. (in Russian), 1974.

Egorov, Yu. A.: Hydrodynamic Model of Tsunami Wave Generated by the Eruption of an Underwater Volcano, in: Natural Catastrophies and Disasters in the Far East Region, edited by: Kaistrenko, V., IMGIG: Yuzhno-Sahalinsk, pp. 95-106 (in Russian), 1990.

Ivanov, V. V. and Korolyov, Yu. P.: Tsunami waves generated by the eruption of underwater volcano, Tsunami Investigations, 1, 45-48 (in Russian), 1986.

Le Mehaute, B.: Explosion-generated water waves, Adv. Hydrosci., N. Y. Acad. Press, 7, 1-79, 1971.

Pelinovskij, E. N.: Nonlinear Dynamics of Tsunami Waves, Institute of Applied Physics, Gorky, 1982.

Pelinovskij, E.: Hydrodynamics of Tsunami Waves, Institute of Applied Physics, Nizhny Novgorod (in Russian), 1996.

Sretenskij, L. N.: Theory of Wave Motion in Fluid. - M., Nauka, 816 pp. (in Russian), 1977.

Taziev, G.: Volcanoes. M., 117 p., 1963. (Russian translation of Haroun Tazieff: Les volcanoes, Paris, 108 p., 1961)

Tinti, S., Bortolucci, E., Chiavettieri, C.: Tsunami excitation by submarine slides in shallow-water approximation, Pure Appl. Geophys., 158(4), 759-797, 2001.

Woods, A. W., Bower, S. M., and Bursik, M. I.: Models of explosive volcanism, Nonlin. Processes Geophys., 2, 269-279, 1995, http://www.nonlin-processes-geophys.net/2/269/1995/. 